\title{
INTELLIGENT BIM-BASED CONSTRUCTION SCHEDULING USING DISCRETE EVENT SIMULATION
}

\author{
Markus König \\ Christian Koch \\ Ruhr-Universität Bochum \\ Chair of Computing in Engineering \\ Universitätsstr. 150 \\ D-44780 Bochum, Germany
}

\author{
Ilka Habenicht \\ Sven Spieckermann \\ SimPlan AG \\ Edmund-Seng-Str. 3-5 \\ D-63477 Maintal, Germany
}

\begin{abstract}
In the last years simulation approaches are increasingly used to support construction scheduling. For that purpose, different kinds of planning data have to be analyzed and integrated to perform realistic and suitable simulation, like building information models, bill of quantities, framework schedules, delivery dates, or available resources. However, a major challenge remains: the efficient specification of realistic and valid interdependencies between construction activities. This specification process is error-prone and often small variations of the input data lead to extensive modifications. This paper presents an intelligent concept to store interdependencies between activities in order to reuse them for handling modifications and different alternatives. Furthermore, the correctness of the interdependencies can be checked and visually highlighted. Finally, a realistic case study is presented to show the advantages of the approach. The approach was developed within the MEFISTO project, supported by the German Federal Ministry of Education and Research.
\end{abstract}

\section{INTRODUCTION}

Simulation is a well-established approach to schedule, analyze, and improve production processes in manufacturing industries. In civil engineering, the application of simulations to support construction scheduling has increased in the last years. This development is caused by the application of Building Information Modeling (BIM) in the planning of construction processes. Planning data stored by BIM can also be used for simulation-based scheduling, like 3D building models, bill of quantities, framework schedules, delivery dates, and available resources. In addition, construction processes and interdependencies between the activities must be specified in order to perform a realistic and suitable simulation. Usually, processes and their interdependencies must be specified manually. This makes the process very errorprone and a small adoption of the building model or the construction method leads to extensive modifications.

Currently, the application of process patterns is a well-established concept to define the construction process. Process patterns and simple interdependency definitions can be reused in the context of different projects. In addition to this, however, a multitude of more complex interdependencies need to be considered regarding technological and strategic aspects. These interdependencies are often very project-specific and can rarely be reused. This paper puts forward an intelligent approach to assign process patterns and to define interdependencies automatically. The presented method uses templates based on linked building information data to handle modifications and different scenarios efficiently. 


\section{RELATED WORK}

BIM includes all processes that define, manage, apply and adapt a digital model of a facility, like a building or an infrastructure. These digital representations, including geometric and functional aspects, are called building information models. Building information models are often used as shared data and knowledge resources to support planning, construction, management, utilization, revitalization, and deconstruction activities (Eastman et al. 2008). The BIM concept allows virtual construction of a facility prior to its actual physical construction, in order to reduce uncertainty, improve safety, work out problems, and simulate and analyze potential impacts (Smith 2007). Three-dimensional geometric objects, like spaces, walls, columns, and slabs, with relations and life-cycle attributes, are defined mainly by means of building information models. These models are often associated with the Industry Foundation Classes (IFCs), which is a data structure for representing complex building information. The IFCs have been developed by buildingSMART as a neutral, non-proprietary and open standard for sharing BIM data (Underwood and Isikdag 2009). Besides IFCs, other partially heterogeneous data formats have been developed to describe further information of a construction project. For example, the German GAEB standard is used to define the bill of quantities in a formal and reusable way, which can also be linked easily to building elements. Furthermore, various BIM software systems exist, that employ individual attributes and specific data formats.

Using BIM for construction management has many benefits. Building information models extended by construction process information are often used to visualize a construction schedule as a 4D visualization. Common practice in this case is to manually assign building elements to construction processes. Many planning tools provide special attributes and rules to simplify this assignment process. However, the underlying schedule needs to be defined beforehand. Recently, an increasing number of concepts have been developed to integrate BIM into construction scheduling itself (Goedert and Meadati 2008). Different planning activities require different kinds of information about the building structure to specify essential input data. BIM-based quantity takeoffs are used to generate input data in order to calculate execution times of construction processes (Zhang and $\mathrm{Hu}$ 2011). On the other hand, building geometry and spatial relations are analyzed to find adequate construction sections and sequences.

Over the past decade, various simulations techniques have been developed and applied to support construction scheduling. In this context, discrete-event simulation concepts are primarily used to simulate and analyze construction operations. For example, simulation models can be created to calculate the utilization of resources or to identify logistics bottlenecks by analyzing transport ways and storage areas. Various simulation tools and frameworks are available to model construction operations. However, the creation of a simulation model is mostly project-specific and reusing models for different projects is generally not possible. Today, this is one of the most important barriers for the implementation of simulation in the construction industry (AbouRizk et al. 2011). Due to the propagation of BIM-related data sources, new concepts are being developed to simplify the definition of simulation models significantly. Building information models can be applied to define essential data for construction simulation. For example, the building geometry can be used to visualize simulation models and results (Kamat and Martinez 2003). Another innovative concept is to integrate building information models into the simulation input data creation process. Exemplarily, Wu et al. 2010 developed a hierarchical process pattern approach for bridge construction. Process patterns are assigned to bridge components considering different levels of details. In this way, construction schedules can be determined by using an abstract simulation model based on the generated processes, including their interdependency and resource constraints.

\section{BIM-BASED CONSTRUCTION SCHEDULING}

In the context of construction scheduling, different planning data sources need to be analyzed, prepared, and integrated. An increasing amount of information is stored in building information models. The efficient usage of building information models depends on the consistency of the relations between different data sources and models. A typical example is the quantity takeoff model. Quantity takeoff data can be 
calculated based on the building elements and the bill of quantities. This, of course, makes relations between building elements and bill of quantities necessary. These relations can be defined by using different planning and estimation tools in a manual or automatic manner. The quantity takeoff data is necessary to determine realistic process durations in construction scheduling. For the presented intelligent BIM-based construction scheduling approach, different relations between the planning data stored in a consistent building information model are required. Therefore, a so-called Multi-Model approach has been developed within the MEFISTO project together with industrial and scientific partners (Scherer and Schapke 2011).

\subsection{Multi-Model Approach}

Normally, different data sources are used to specify extensive building information models. In our case, building information models comprise more than one data file or database. Consequently, a building information model is a multi-model. A multi-model integrates different data sources by using external links based on a separate link model (Scherer and Schapke 2011). The original data sources are not modified. Only references to the BIM elements are stored in the link model. A typical example is the linkage between building elements and framework schedules, which are a basis for construction scheduling. Thereby, geometrical information, activities, and timetables are linked and can be used for 4D animations.

In the MEFISTO project, a flexible linking approach based on simple text files has been developed. Individual multi-models can be used for different planning tasks. Multi-models can be defined for cost estimation, risk management, and construction simulation. In Figure 1, a multi-model container for simulation-based construction scheduling is highlighted. This multi-model container comprises data models of the building elements (IFC), bill of quantities (BoQ), quantity takeoff (QTO), resources (RES), and framework schedules (FS). All these different models are provided as XML, CSV, or STEP ASCII files. The linkage can be done semi-automatically or manually. In the MEFISTO project, an extended version of the software RIB iTWO is used to generate multi-models.

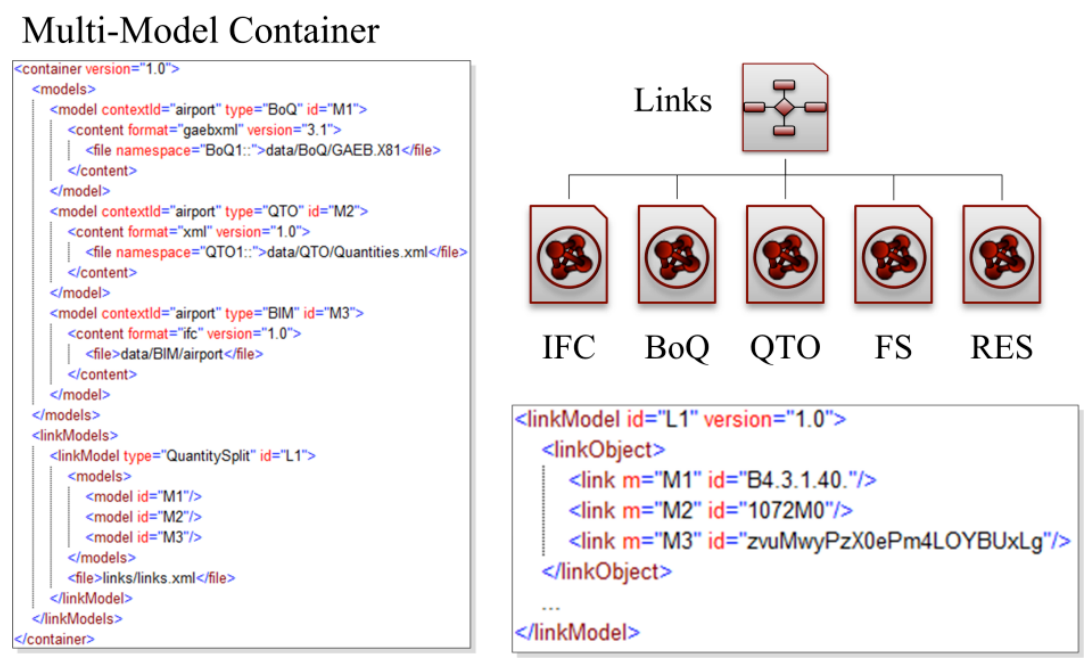

Figure 2: Multi-model for construction scheduling

\subsection{Process Pattern}

The specification of construction processes is the most essential task for scheduling. In practice, different construction methods exist to construct a certain element of the building structure. For example, the construction of an in-situ concrete wall normally consists of erecting the formwork, installing the reinforcement, placing the in-situ concrete, curing the concrete, and stripping the formwork. These processes can be used for similar building elements. In the presented approach, the processes of a construction method 
are modeled as a process pattern. Consequently, process patterns are reusable construction methods which are stored in a pattern catalog. For each process, attributes can be specified, e.g., performance factors dependent on the bill of quantities, required resources, and needed material. Various technological interdependencies exist between the processes of a construction method. In the context of this approach, the interdependencies between processes of a process pattern are called local interdependencies. In Figure 3, a typical process pattern with local interdependencies to construct in-situ concrete walls is shown.

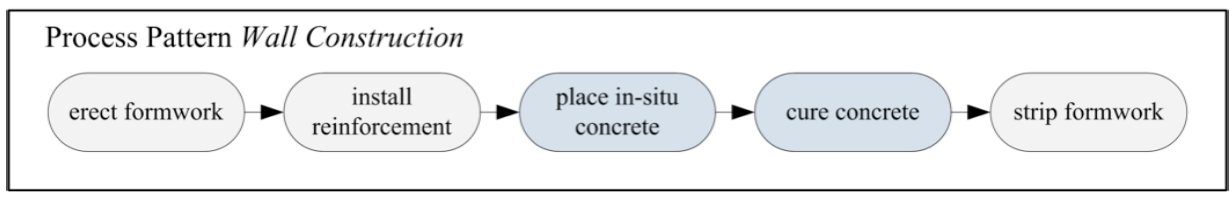

Figure 3: Process pattern wall construction

The construction process specification is done in the following way: either a single process pattern or several process patterns are assigned to the building elements. This means that the process patterns instantiate individual processes for each element. The multi-model is used to select the building elements for the process specification. Consequently, an individual process is linked to the building element so that information about the bill of quantities and the quantity takeoff can be determined to calculate the process duration based on the performance factors.

\subsection{Modeling Complex Interdependencies}

Normally, several hundreds or thousands of construction processes must be defined for each construction project. A multitude of technological and strategic interdependencies between the construction processes need to be considered. In addition to local interdependencies, many complex dependencies between building elements of different types, sections or levels exist. These interdependencies are called global interdependencies in the context of the presented approach. Global interdependencies are not stored within a process pattern and strongly depend on the actual project conditions and the building design. In Figure 4, the construction processes and local interdependencies of the in-situ concrete walls of a concreting section are shown. The local technological interdependencies are modeled separately for all construction processes.

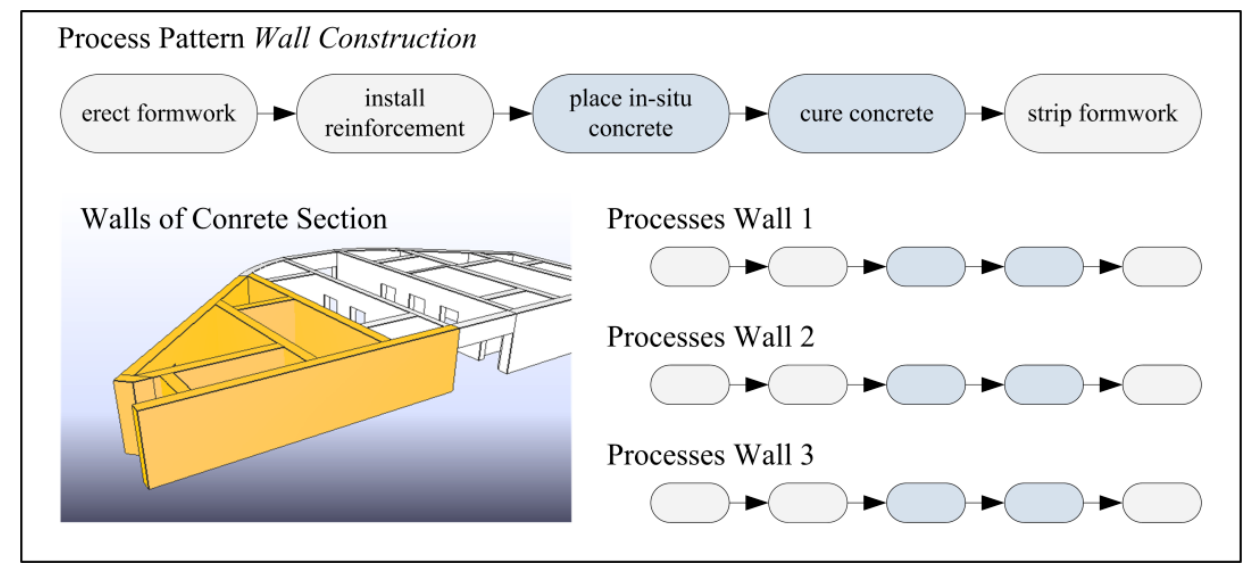

Figure 4: Interdependencies between wall construction processes

A possible construction strategy is to concrete all walls in one single concreting process by using a concrete pump. Consequently, additional strategic interdependencies need to be defined as shown in Figure 5. 
Now, the main challenge is to define intelligent concepts so that complex interdependencies can be reused for different concreting sections without extensive and time-consuming manual modifications.

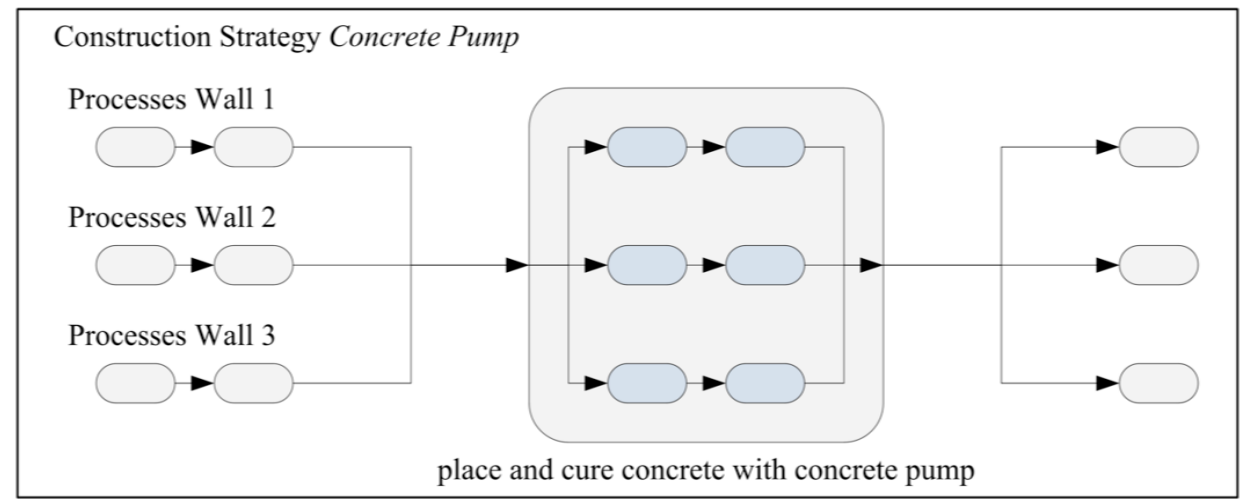

Figure 5: Global interdependencies between wall construction processes with regards of using a concrete pump

Obviously, the interdependency specification can be very error-prone, which can lead to incorrect process sequences. In this context, incorrectness means that the resulting process graph contains cycles or duplicated processes. For this reason, different correctness and consistency checks have been implemented to guarantee adequate processes and interdependencies. During specification, construction processes are continuously checked for process patterns that are assigned several times to the same building elements and whether new interdependencies would result in execution cycles. Furthermore, the process graph can be visualized and inspected manually (cf. Figure 6).

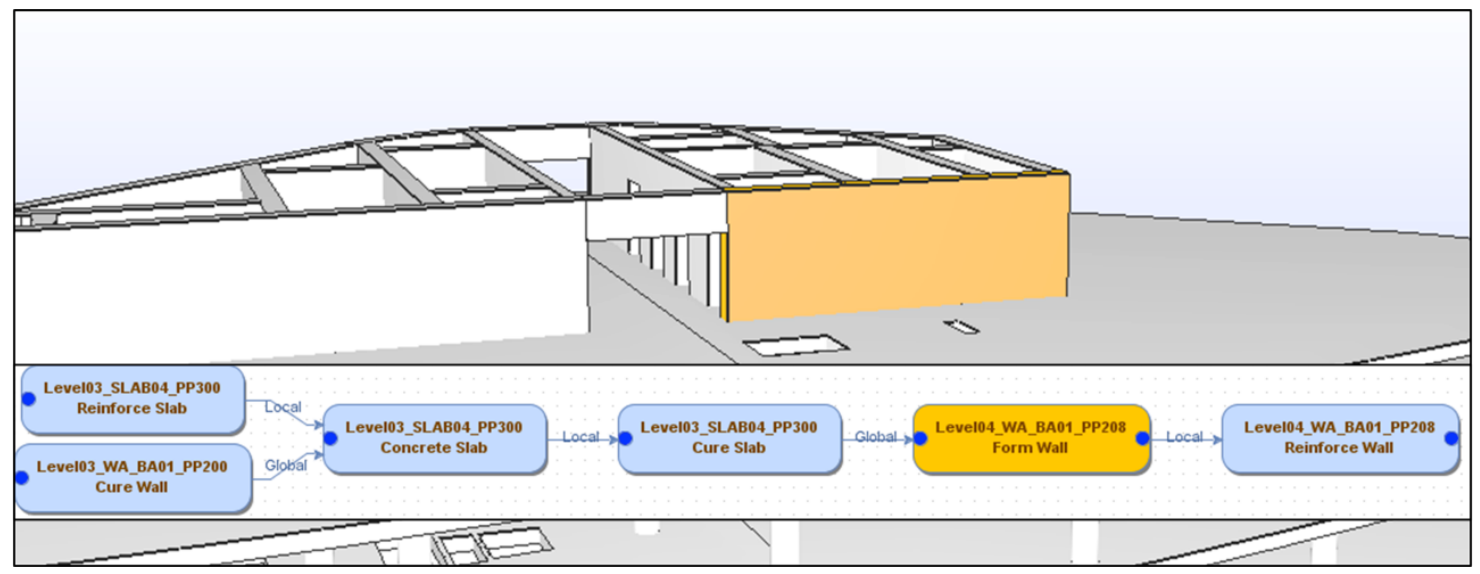

Figure 6: Visualized local and global interdependencies

\subsection{Constraint-based Simulation}

In this paper, construction simulation is applied to generate a valid schedule based on the specified processes and interdependencies with regard to available resources and working shifts. An extended discreteevent simulation concept is used, taking into account constraint satisfaction (König et al. 2007). This socalled constraint-based simulation approach can be used to simulate construction processes in a flexible manner. Constraint-based simulation means that each time an event occurs, the process and resource constraints are checked to identify which processes can be started. That means, process interdependencies and resource availability are considered as constraints. In consequence, a process can be started in the course of a simulation if all associated predecessor processes are completed and the required resources are 
available. An integration concept has been developed to import, analyze and evaluate the input data for constraint-based simulation of construction processes. As a result, the data of the multi-model as well as the specified processes and their interdependencies can easily be used and transferred.

\section{REUSING PROCESS AND INTERDEPENDENCY DEFINITIONS}

Using integrated BIM data and flexible process patterns are significant steps to increase the efficiency of defining processes and interdependencies. Another important aspect is that complex interdependency definitions can be reused for different construction versions, scenarios, and projects without extensive modifications. Reusing existing information can be achieved by using dynamic and configurable templates. Templates are blueprints to generate individual processes and interdependencies for a specific project depending on actual BIM data.

\subsection{Process Pattern Assignment Templates}

The first template category is the assignment of process patterns to building objects in order to generate individual construction processes. For example, the assignment of the process pattern "in-situ concrete wall construction" to every building element which is of the type "IfcWall" can be modeled as a simple template. More specific templates take into account various attributes of different data models by analyzing the existing model links. Consequently, a query language is needed to select the correct building elements for assignment. Different general and specific query languages are already available. The Structured Query Language (SQL) is a very powerful query language for relational databases. In this context, a query is a template to describe which attribute values of a relation are included in the final result. The resulting data can be restricted by using complex clauses. Another query language for selecting partial model data is the Partial Model Query Language (PMQL) developed by VTT Building and Transport (Adachi 2002). PMQL itself is XML data. Therefore, it can be used very flexible. PMQL also contains conditional expressions based on SQL. PMQL was first used in the context of an IFC model server.

In the presented approach, the PMQL syntax is used to specify building element identifiers for defining reusable process pattern assignment templates. In general, the complete multi-model, including all links, must be taken into account for selecting building elements. However, only selective attributes of certain data objects described by the multi-model can currently be used. Figure 7 shows a typical template to assign a process pattern to some building elements in a certain storey. An extended PMQL interpreter is under development to generate individual construction processes for the selected building elements.

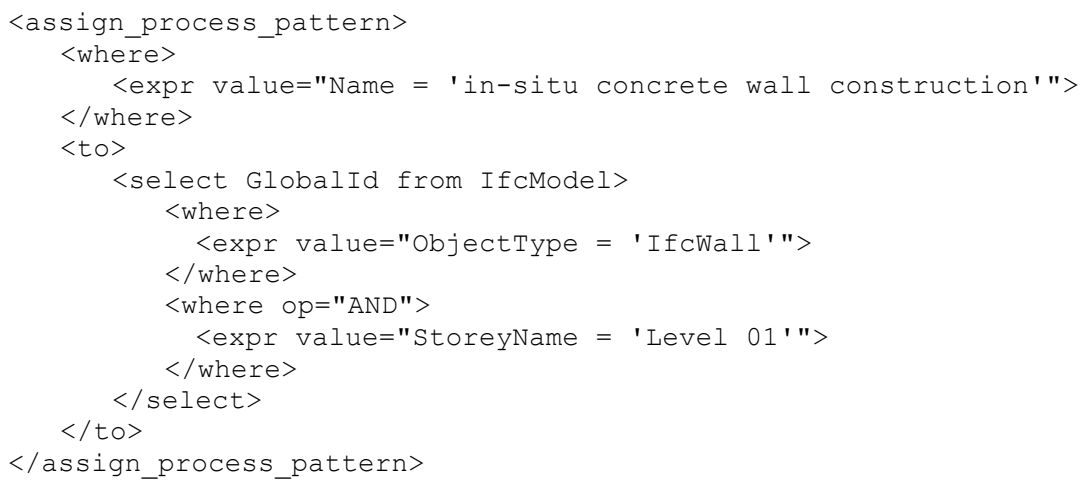

Figure 7: Process pattern assignment template for in-situ concrete wall construction

\subsection{Interdependency Templates}

Special templates have been formalized for the generation of individual interdependencies between processes of different building elements and process patterns. Interdependency templates strongly depend on 
the considered construction project. However, various templates exist which can be used in a more general manner. A typical interdependency template is, for example, that the slabs of a certain storey cannot be constructed before all columns of the lower storey are load-bearing (cf. Figure 8). Thereby, information about process patterns and building elements must be taken into account to formulate such interdependency templates. Therefore, a special assigned-to clause was introduced. With this clause, a selection can be defined based on the assigned building elements of a certain process pattern.

Interdependency templates are often specified by bearing in mind topological or geometrical aspects. For example, only a certain amount of elements or processes of a work section have to be selected. Therefore, it is necessary to extend the query language by topological and spatial properties. At the moment, only grouping aspects can be considered. This means that certain building objects can be assigned to different groups. Groups can be defined manually or by selecting certain attributes. For example, concreting sections are often modeled in the context of framework scheduling. This information can be used to define interdependencies between concerting processes.

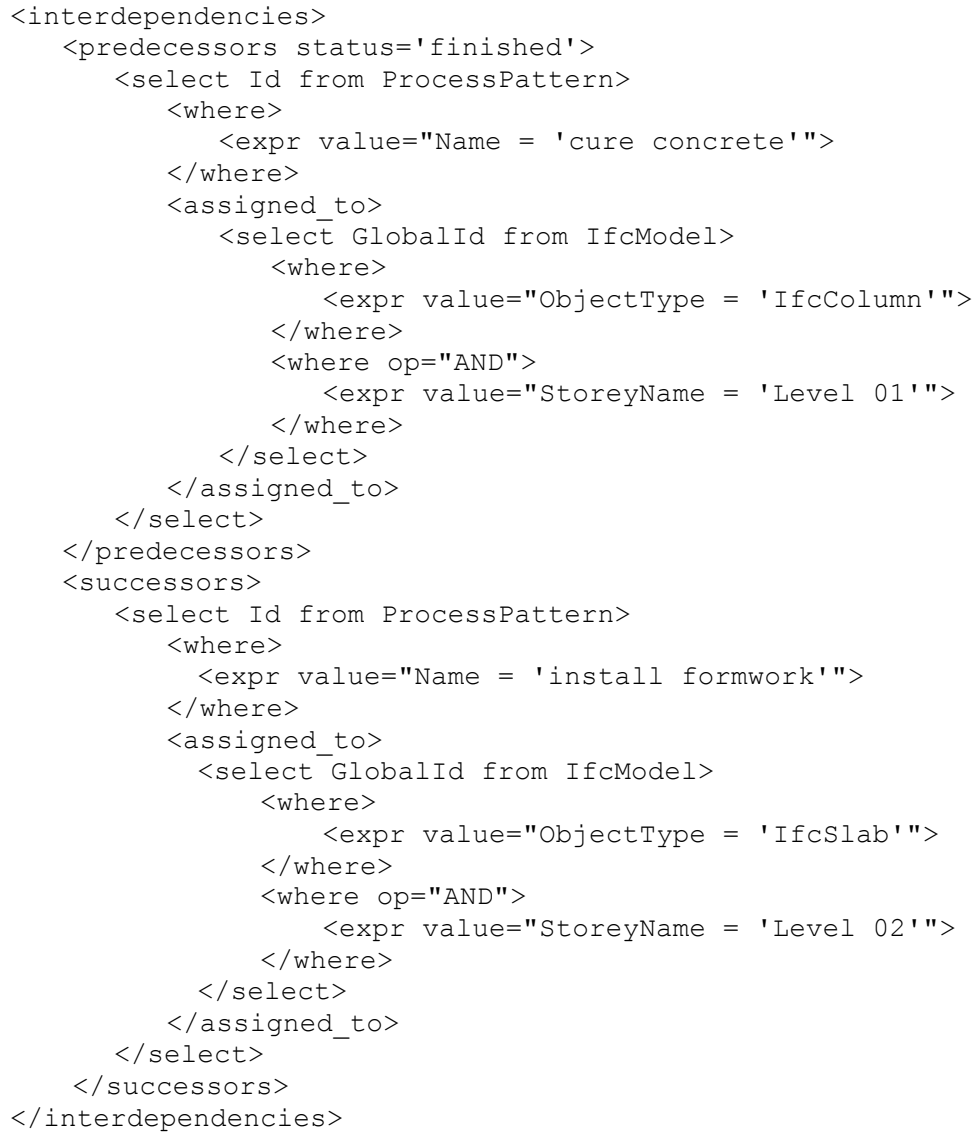

Figure 8: Interdependency template for slab and column construction processes

In the future, spatial queries will also be considered. Spatial queries describe, for example, the selection of building elements which are inside in a zone or which lie between axes. Different approaches exist to formulate topological and spatial queries. Tulke et al. 2008 presented a concept to decompose BIM objects for scheduling. Building objects can be selected and decomposed by using a query language. A more general spatial language for the topological analysis of building models is described in Borrmann and Rank 2009. Here, complex spatial queries can be modeled and interpreted by hierarchical structures. 


\subsection{Handling Design Modifications}

Assigning process patterns and generating interdependencies by using templates also helps to handle project modifications like design or construction changes. Generally, templates should be defined based on selected attributes of the underlying data models and should not consider exact identifiers of the building elements. In consequence, it is possible to apply, for example, a process pattern assignment template without explicit knowledge of the exact amount of building elements. New processes could be generated automatically while the process patterns and the templates are still valid if the building design was changed, e.g., by adding new walls or columns. The same principle applies to the interdependency templates. However, the construction manager must check the consistency and correctness of all processes and independencies after each modification. Therefore, it is very important to provide intelligent and adequate control mechanisms. Simple control mechanisms are to highlight new or removed processes and interdependencies, as well as functionalities to check the resulting interdependency graph.

\subsection{Scenario Management}

Another advantage of using flexible templates is the efficient specification of different construction scenarios. Normally, different construction methods and execution strategies can be applied to define building structures. For example, a certain building can be constructed by using precast concrete elements instead of in-situ concrete or by applying climbing formwork systems in place of standard formwork elements. Various possibilities exist for the execution of construction works. However, only a restricted amount of construction methods and execution strategies are possible, efficient, and manageable for a certain project.

Construction simulation can be applied very efficiently to analyze different scenarios. Due to the fact that the preparation of input data for construction simulation is very challenging, the investigation of alternative construction methods or execution sequences is not very common. This drawback can be overcome by applying reusable patterns to define the necessary input data for construction simulation. For these reasons, a flexible scenario management is under development in order to define adequate simulation input data based on the presented pattern-based approach.

Normally, the specification of a construction scenario comprises the adaption of process patterns, resources, shifts, building element groups, process pattern assignment templates, and interdependency templates. Certain specifications can be reused for various scenarios. For that purpose, the simulation input data is stored in separate data files to enable an easy adoption. Subsequently, for each scenario the input data, which is primarily processes and interdependencies of the construction works, can be generated. The results of each simulation scenario are individual schedules with regard to the available resources and shifts. For finding an efficient and realistic schedule, the resulting schedules must be compared in an adequate manner, taking into account the given project restrictions. The scheduling foundations and results can be archived for a transparent decision support. Furthermore, these scheduling results can be reused in future projects.

\section{CASE STUDY}

The highlighted concepts have been validated extensively within the MEFISTO project. In this paper, a case study is presented, which comprises the simulation-based scheduling of shell construction works of a real office building with nineteen similar storeys. The shell construction processes are specified by applying certain process pattern assignments and interdependency templates. The preparation steps are done by using a BIM-based scheduling tool, the so-called SiteSimEditor. The SiteSimEditor is an application based on the Open IFC Tools (OpenIFCTools 2012) for reading and analyzing multi-models as well as for defining, managing, and applying process patterns and templates for construction simulation. The Siemens PLM software Plant Simulation by Siemens PLM Software, version 9.0, was used as the simulation engine. The constraint-based simulation models were developed by integrating and adapting the Sim- 
ulation Toolkit Shipbuilding (STS) of the Simulation Cooperation in the Maritime Industries (SimCoMar, Steinhauer 2010). Further information can be found on the SimCoMar website (SimCoMar 2012).

The case study applies an alternative construction method to build the in-situ concrete columns of the office building. First of all, standard process patterns and templates for constructing columns, slabs, beams, and walls of the building have been defined and applied. The process pattern for column construction consists of five processes shown in Figure 9a.

a) Process Pattern Column Construction

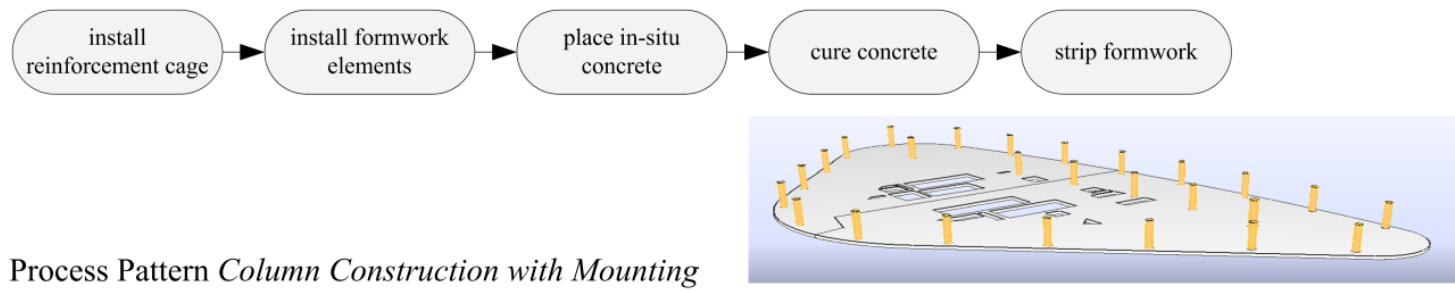

b) Process Pattern Column Construction with Mounting

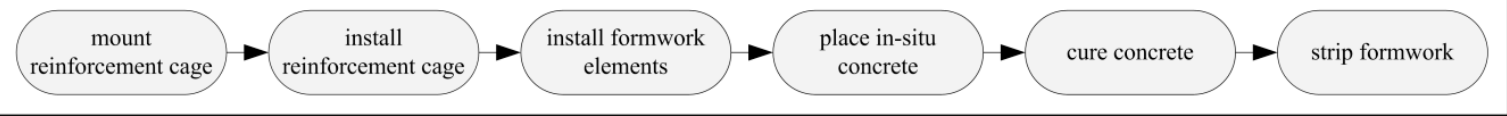

Figure 9: Interdependency template for slab and column construction processes

Thereby, the process "install reinforcement cage" requires a crane to hold the reinforcement cage continuously until the process is finished. Only one tower crane is available for this project. Based on these specifications, a construction schedule was generated by using constraint-based simulation. The schedule shows that the tower crane is highly utilized. Consequently, the duration to construct one storey was higher than expected. However, an essential planning condition was that the shell construction works of a storey should be finished within 23 work days. Therefore, an alternative process pattern for column construction was specified to reduce the construction time. The alternative process pattern consists of six processes (cf. Figure 9b). The original process "install reinforcement cage" was divided into two processes. Within the process "mount reinforcement cage" a crane is used to transport and hold the reinforcement cage while workers are attaching the cage to an additional mounting element. Afterwards, the final reinforcement installation process can be carried out without using a crane. The alternative processes for all columns were easily generated by applying an alternative process pattern assignment template. By analyzing the new simulated schedule, it could be shown that the given time restriction was satisfied by applying the alternative construction method for column erection. Both scenarios can be visualized and compared by using the SiteSimEditor (cf. Figure 10).

\section{CONCLUSIONS AND OUTLOOK}

Normally, the specification of realistic processes and interdependencies for construction simulation is very time-consuming. Therefore, complex planning data must be analyzed, interpreted and prepared. Building information models are increasingly used as a basis for planning and scheduling. In this paper, an intelligent and efficient approach is presented to generate input data for construction simulation by using linked building information data and reusable templates. Two general template types are highlighted. Process pattern assignment templates can be used to automatically assign process patterns to building elements to generate individual construction processes. Many interdependencies exist between the construction processes, which are very important in the context of scheduling. By using interdependency templates, the effort to define individual interdependencies between processes can be reduced. The highlighted templates can be stored based on a formal definition language. The definition language is based on the Partial Model Query Language (PMQL). 
Future research will deal with extensions of the definition language to specify more flexible and complex templates. For example, spatial relationships can be taken into account or other properties of the building information models can be used. Another research topic is the automatic identification of templates based on individual processes and interdependencies.

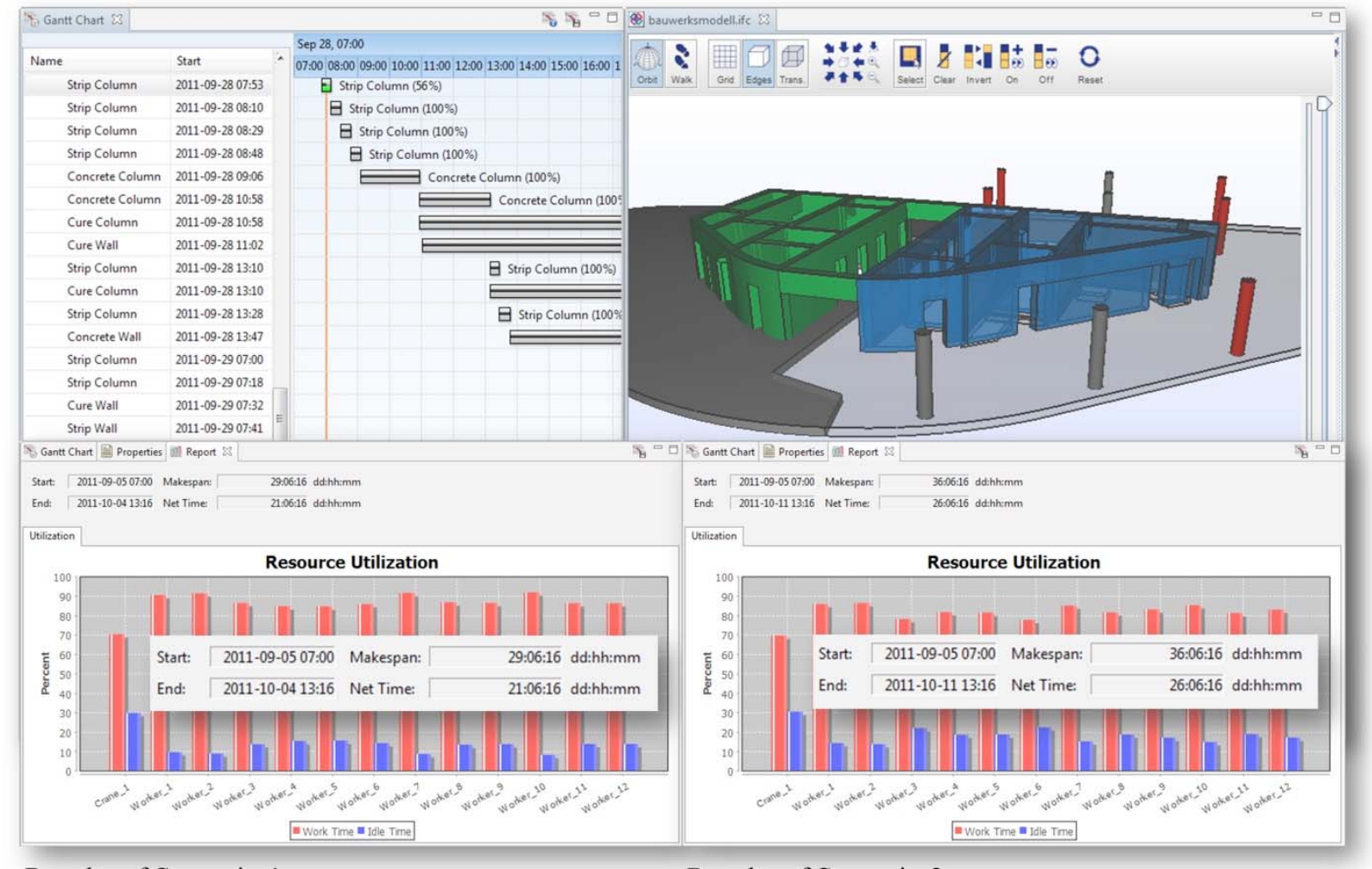

Results of Scenario 1

Results of Scenario 2

Figure 10: Visualization and animation of simulation results in the SiteSimEditor

\section{ACKNOWLEDGMENTS}

The authors gratefully acknowledge the financial support of the German Federal Ministry of Education and Research for the MEFISTO project. The authors also give profuse thanks to the Open IFC Tools team.

\section{REFERENCES}

AbouRizk, S., D. Halpin, Y. Mohamed, and U. Hermann. 2011. "Research in Modeling and Simulation for Improving Construction Engineering Operations." Journal of Construction Engineering and Management 137 (10): 843-852.

Adachi, Y. 2002. "Overview of Partial Model Query Language - VTT-TEC-ADA-12.” SECOM Co., Ltd. / VTT Building and Transport. Accessed May 23, 2012. http://cic.vtt.fi/projects/ifcsvr/tec/VTT-TECADA-12.pdf.

Borrmann, A., and E. Rank. 2009. "Specification and implementation of directional operators in a 3D spatial query language for building information models." Advanced Engineering Informatics 23 (1): 32-44.

Eastman, C., P. Teicholz, R. Sacks, and K. Liston. 2008. BIM Handbook: A Guide to Building Information Modeling for Owners, Managers, Designers. New Jersey: Wiley \& Sons, Inc. 
Goedert, J. D., and P. Meadati. 2008. "Integration of construction process documentation into Building Information Modeling." Journal of Construction Engineering and Management 134 (7): 509-516.

Smith, D. 2007. "An Introduction to Building Information Modeling (BIM)." Journal of Building Information Modeling. Accessed May 23, 2012. http://www.wbdg.org/pdfs/jbim_fall07.pdf.

Kamat, V. R., and J. C. Martinez. 2003. "Validating complex construction simulation models using 3D visualization." Systems Analysis Modelling Simulation 43 (4): 455-467.

König, M., U. Beißert, D. Steinhauer, and H.-J. Bargstädt. 2007. "Constraint-Based Simulation of Outfitting Processes in Shipbuilding and Civil Engineering." In Proceedings of the 6th EUROSIM Congress on Modeling and Simulation, CD-ROM. Ljubljana.

OpenIFCTools. 2012. "Open IFC Tools Processing / Visualization / 4D." Accessed May 23, 2012. http://www.openifctools.org.

Scherer, R., and S.-E. Schapke. 2011. "A distributed multi-model-based management information system for simulation and decision making on construction projects." Advanced Engineering Informatics 25 (4): 582-599.

Steinhauer, D. 2010. "GeneSim - Development of a Generic Data Model for Production Simulation in Shipbuilding." In Proceeding of 9th European Conference on IT Application in the Maritime Industries (COMPIT), 304-310. Gubbio (Italy).

SimCoMar. 2012. "Simulation Cooperation in the Maritime Industries." Accessed May 23, 2012. http://www.simcomar.com.

Tulke J., M. Nour, and K. Beucke 2008. "Decomposition of BIM objects for scheduling and 4D simulation." In Proceedings of the 7th European Conference on Product and Process Modelling in the Building and related Industries, Edited by Zarli, A., and R. Scherer. Sophia Antipolice (France).

Underwood, J., and U. Isikdag. 2009. Handbook of Research on Building Information Modeling and Construction Informatics: Concepts and Technologies. New York: Information Science Publishing.

Wu, I.-C., A. Borrmann, U. Beißert, M. König, and E. Rank. 2010. „Bridge construction schedule generation with pattern-based construction methods and constraint-based simulation." Advanced Engineering Informatics 24 (4): 379-388.

Zhang, J.P., and Z.Z. Hu 2010. "BIM- and 4D-based integrated solution of analysis and management for conflicts and structural safety problems during construction: 1. Principles and methodologies." Automation in Construction 20 (2): 155-166.

\section{AUTHOR BIOGRAPHIES}

MARKUS KÖNIG is professor of Computing in Engineering at Ruhr-University Bochum, Germany. Previously, he was assistant professor of Theoretical Methods for Project Management at BauhausUniversity Weimar, Germany. He obtained his Ph.D. in Civil Engineering from Leibniz-University Hanover, Germany in 2003. His research interests include construction simulation, building information modeling, heuristic optimization techniques, knowledge management in construction, intelligent computing in engineering and computational steering. His email address is koenig@inf.bi.rub.de.

ILKA HABENICHT is consultant at SimPlan AG, Maintal, Germany. She received her diploma at Industrial Engineering from the Technical University Ilmenau and her doctorate in Business Informatics from the FernUniverstiät in Hagen. She has been participating in simulation projects for several industries. Her email address is ilka.habenicht@simplan.de.

CHRISTIAN KOCH is a postdoctoral group leader at the chair/institute of Computing in Engineering at the Ruhr-Universität Bochum. In 2008, he received his doctorate in Civil Engineering from the BauhausUniversität Weimar for his work on distributed building information modeling. Since 2010, he leads the Computer Vision and Mixed Reality group that conducts research on vision-based IT support in several 
fields of construction, such as progress monitoring, condition assessment and facility maintenance. His email address is koch@inf.bi.rub.de.

SVEN SPIECKERMANN, Ph.D., is Chief Executive Officer at SimPlan AG, Maintal, Germany, mainly working as a senior consultant and project manager in simulation projects for several industries. Since 1992, he has been participating in over 200 simulation projects and various joint research initiatives. Additionally, he has been giving lectures in simulation at the Technical University of Braunschweig since 1995 and at the Technical University of Darmstadt since 2008. He has published several papers on simulation, simulation-based optimization and related topics. His email contact is sven.spieckermann@simplan.de. 